\title{
Neuland: Praxis-Leitlinien gemeinsam mit Schmerz-Patienten entwickeln
}

Die Deutsche Gesellschaft für Schmerztherapie e.V. und die Patientenorganisation Deutsche Schmerzliga e.V. betreten Neuland bei der Entwicklung evidenzbasierter Praxis-Leitlinien für die Diagnostik und Therapie chronischer Schmerzen. Auf einer freigeschalteten Online- Plattform "dgs-praxisleitlinien.de" konnten nicht nur Schmerzmediziner, sondern auch Angehörige anderer Heilberufe und vor allem Patienten und deren Angehörige die Leitlinien sichten, prüfen und kommentieren. Dazu wurden die ersten zur Kommentierung vorliegenden Leitlinien in eine auch für Nichtmediziner verständliche Sprache übersetzt.

Die beiden ersten PraxisLeitlinien der Deutschen Gesellschaft für Schmerztherapie zu „Tumorschmerzen“ und "tumorbedingten Durchbruchschmerzen" konnten von Fachkreisen und Patienten kommentiert werden.

In fortgeschrittenen Stadien ihrer Erkrankung leiden 50 bis 70 Prozent der Krebspatienten an starken Schmerzen. »Punktuell hat sich die Versorgung der Patienten in den letzten zwei Jahrzehnten zwar verbessert, doch nach wie vor gibt es eklatante Defizite in der Versorgung ", konstatiert Dr.med. Dipl. Psych. Johannes Horlemann, Sprecher des Arbeitskreises Palliativmedzin und Vizepräsident der Deutschen Gesellschaft für Schmerztherapie.

Doch
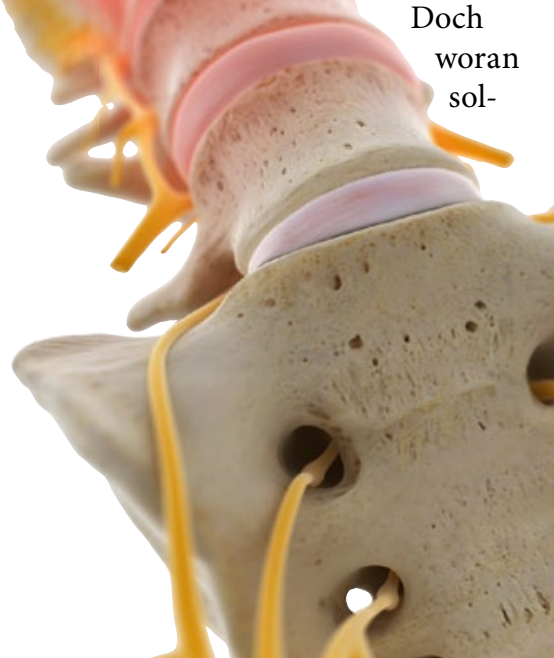

len und können sich Ärzte bei der Behandlung von Tumorschmerzen orientieren? Vorhandene Leitlinien, kritisieren Horlemann und seine Kolleginnen und Kollegen von der Fachgesellschaft, sind nicht mehr aktuell, kollidieren mit wirtschaftlichen Vorgaben oder sind schlicht in der Praxis nicht umsetzbar. Hinzu kommt, dass sich die Leitlinien zum Tumorschmerz vornehmlich auf Studien mit Schmerzmedikamenten stützen. "Dies greift zu kurz und verstößt gegen den multimodalen Anspruch einer modernen Schmerzmedizin und entspricht nicht dem multidimensionalen Leid des betroffenen Patienten“, sagt Dr. med. Gerhard H.H. Müller-Schwefe, Präsident der Deutschen Gesellschaft für Schmerztherapie e. V. Darum hatte die Mitgliederversammlung der Deutschen Gesellschaft für Schmerztherapie beschlossen, praxisnahe Leitlinien im Bereich der Schmerzmedizin zu entwickeln.

Dabei geht die Fachgesellschaft zusammen mit der Deutschen Schmerzliga gleich in mehrfacher Hinsicht neue Wege: Sie integriert und bewertet bei der Entwicklung der Praxis-Leitlinien nicht nur Daten aus der vorhandenen wissenschaftlichen Literatur, sondern räumt dem Wissen und der Erfahrung von Angehörigen anderer Heil- und Pflegeberufe und vor allem der Sichtweise der Patienten einen hohen Stellenwert ein.

Die beiden Schmerzorganisationen wollen durch ihre Leitlinienarbeit neue Maßstäbe für eine patientenorientierte Schmerzmedizin setzen. „Es gilt“, so Dr. Überall, „für die Versorgung von Schmerzpatienten in der täglichen Praxis Behandlungsspielräume und Perspektiven zu öffnen, um den Patienten genau das wieder zurück zu geben, was sie im Kampf mit ihren chronischen Schmerzen häufig verloren haben: Lebensfreude und Lebensqualität.“

Weitere Informationen finden Sie unter: http://www.dgs-praxisleitlinien.de

\section{Frühe Diagnose von Hautkrebsmetastasen}

Bei Patienten mit primären Melanomen, dem sogenannten „schwarzen Hautkrebs", können mittels eines neuen Diagnoseverfahrens Metastasen besser erkannt werden. In der nun vorliegenden Studie wurde der Einsatz der Hybrid Single-Photon-Emissions-Computer-Tomographie - eines Bildgebungsverfahrens mittels Computertomographie (SPECT / CT) im Vergleich mit der Standarddiagnoseüberprüft.

Es konnte belegt werden, dass nach einem Zeitraum von ca. 28,8 Monaten Patienten, bei denen diese neue Diagnosemethode angewandt wurde, eine deutlich niedrigere Rückfallrate aufwiesen als Patienten, die mit dem "alten" Standard-Verfahren untersucht wurden.

Stoffels I et al. - Free Survival in Melanoma. JAMA. 2012;308(10):1007-1014. doi:10.1001/2012. jama.11030.

\section{HPV: Tumorzellen werden \\ strahlensensibler}

Humane Papillomaviren (HPV) können u.a. Krebserkrankungen in Gebärmutterhals und im Mund-Rachen-Bereich hervorrufen. Diese Tumoren reagieren offenbar deutlich empfindlicher auf eine Strahlentherapie als gleiche Tumoren, in denen keine Viren nachweisbar sind. Diese Erkenntnisse könnten zukünftig für eine individuell angepasste Behandlung genutzt werden. Denselben Effekt beobachtete man bei Gebärmutterhalskrebs. Eine Studie mit Patientinnen, die als Erstbehandlung eine Strahlentherapie erhalten hatten, ergab: Frauen, die positiv auf HPV getestet wurden, hatten ebenfalls eine um 30 Prozent höhere Überlebenschance als Frauen mit HPV-negativen Tumoren.

Lindel K, et al Int. J. Radiat. Biol. Phys. 2006;65(3):769-765. 